\title{
Sensitivity of instrumental responses to an upshift in water deprivation
}

\author{
MATÍAS LÓPEZ and CONCEPCIÓN PAREDES-OLAY \\ Universidad de Oviedo, Oviedo, Spain
}

\begin{abstract}
The role of incentive learning in instrumental performance following an upshift in the degree of water deprivation was analyzed in three experiments. In Experiments $1 \mathrm{~A}$ and 1B, rats trained to perform an instrumental action reinforced by either sucrose or maltodextrin solutions when in a low-deprivation state were shifted to a high-deprivation state and tested in extinction. This shift in water deprivation increased performance only if the animals had been exposed to the reinforcer in the high-deprivation state prior to testing. In Experiment 2, the role of the instrumental contingency in mediating the preexposure effect observed in the first two studies was examined by training rats to make two instrumental actions for different outcomes. The preexposure experience with the outcomes produced a relative increase in performance of the action reinforced with the incentive preexposed in the high-deprivation state when a choice between the two response alternatives was conducted in that state. These experiments support the conclusion that instrumental performance following revaluation of the reinforcer by an upshift in the level of thirst depends on a process of incentive learning.
\end{abstract}

The incentive learning theory proposed by Dickinson and Balleine (1994) suggests that goal-directed behavior is accomplished by the interaction of two separate learned associations. First, instrumental actions are controlled by an expectation of the rewarding properties that will result from an action (i.e., the action-outcome relation acquired during training). Second, through a process of incentive learning, interoceptive properties of deprivation predict that the rewarding properties of a particular outcome will be enhanced in that state. Therefore, in this model, motivational states are claimed to determine directly the incentive value of an outcome and, as a result of incentive learning, to gain conditional control over the incentive value assigned to that outcome, not over instrumental performance directly (see Dickinson, 1997, and Diekinson \& Balleine, 1994, for reviews).

Recent and compelling evidence for incentive learning theory comes from studies of posttraining shifts in primary motivation. For example, Balleine (1992) analyzed the influence of a posttraining increase in the degree of food deprivation on the performance of an instrumental action in extinction. In this study, rats were trained to press a lever for a novel food reward while they were food sated, and then were tested in extinction conditions while they were food deprived. It was found that shifting animals into a state of food deprivation had no detectable effect on the rate of responding. However, if animals were simply exposed to the novel food while in the state of food depri-

This research was supported by grants from the Spanish Ministerio de Educación y Cultura to $\mathrm{M}$. López (Dirección General de Investigación Científica y Técnica, PB96-0561) and to C. Paredes-Olay (Becas de FPI-AP93). Correspondence should be addressed to M. López, Departamento de Psicología, Universidad de Oviedo, Plaza de Feijoo s/n, 33003-Oviedo, Spain (e-mail: mlopez@correo.uniovi.es). vation before testing under the same conditions, then the rate at which rats responded was significantly greater than that for animals that were not preexposed to the food while hungry. The interpretation offered for these findings was that animals have to learn about the new incentive value of the outcome in the shifted state, and that in the absence of the opportunity to learn about the value of the new food under conditions of food deprivation, instrumental performance is controlled only by the previously learned value of the outcome (Balleine, 1992). This process is similar to Tolman's (1949a, 1949b) claim that the changes in the incentive quality of a reinforcer influence instrumental performance, but only through the development of a "cathexis"--that is, through directly experiencing and learning about the changed value of the reinforcer.

It should be noted that this role for incentive learning in instrumental performance following a shift in motivational state is not confined to upshifts in food deprivation. The same pattern of results was also found by Balleine (1992; see also Balleine \& Dickinson, 1994) for the opposite shift - that is, where rats were trained to leverpress for food pellets when food deprived and then tested when undeprived. In this case, rats reduced their performance only when food deprivation was reduced if they were allowed to consume the instrumental outcome when undeprived prior to the test. This finding suggests that just as animals have to learn about the high incentive value of the food pellets when hungry, so too they have to learn about its low value when they are sated.

The role of incentive learning in control of instrumental action has also been confirmed for a posttraining reduction in water deprivation (López, Balleine, \& Dickinson, 1992). In this study, rats trained to perform an instrumental action reinforced with either sucrose or maltodextrin solutions when in a high-deprivation state were subse- 
quently shifted to a low-deprivation state and tested in extinction. This downshift in water deprivation reduced instrumental performance only when animals had been allowed prior experience with the reinforcer while satiated, thus enabling them to learn about its low value in that state. In accord with incentive learning theory, the rats had to learn that the incentive value of the instrumental outcome had changed following the reduction in water deprivation before this motivational shift acted to modify performance. These authors were also able to confirm that this incentive learning effect depended on the instrumental contingency. They trained thirsty rats to perform two instrumental actions with one action earning access to sucrose and the other access to a maltodextrin solution. Prior to this training, however, all the animals had received access to one of the outcomes when nondeprived and to the other following water deprivation. When the animals were given a choice between the two actions in extinction while undeprived, they showed a selective reduction in the performance of the action reinforced with the incentive preexposed in the undeprived state. These findings support the conclusion that motivational control of an instrumental action following a downshift in the level of thirst depends on the animal's knowledge of the action-outcome relation and the current incentive value of that outcome.

The aim of the experiments in this article was to investigate the influence of a posttraining increase in water deprivation on the performance of an instrumental action in extinction in an attempt to assess the generality of incentive learning in within-state motivational shifts. The previous experiments by López et al. (1992) demonstrated that the performance of an instrumental action following a posttraining shift from a high to a low level of water deprivation reduces performance only when the animals have been exposed to the reinforcer in the lowdeprivation state prior to testing. In view of this finding, one obvious question that arises is whether incentive learning is necessary for a shift in the opposite directionthat is, from a low to a high level of water deprivationto affect instrumental performance.

\section{EXPERIMENTS 1A AND 1B}

In these experiments, rats were initially preexposed to an alternating schedule of low and high levels of water deprivation. During this period, half the subjects were given exposure to the reinforcer on days on which they were sated, and the remainder received presentations of the reinforcer while they were water deprived. Following this stage, all animals were trained to press the lever for the reinforcer while in the low-deprivation state. After the final training session, half of the animals that had been preexposed under thirst and half that had been preexposed when they were sated were maintained on free water, while the remainder of the subjects were again deprived of water. Leverpress performance was then assessed in a single extinction test. If the motivational control of performance is mediated by a process of incentive learning, then leverpressing by animals trained in the low-deprivation state and shifted to the high state on test should persist at a level similar to that of animals tested when undeprived, unless they were previously allowed to consume the reinforcer in the high-deprivation state. By contrast, if instrumental performance is a simple function of the degree of deprivation, as the general-drive hypothesis assumes (e.g., Hull, 1943), then extinction performance of both the groups tested when deprived should be greater than that of the groups tested when undeprived.

In Experiments $1 \mathrm{~A}$ and $1 \mathrm{~B}$, the procedures were the same, except that the instrumental reinforcer used in each case differed so that we could examine the generality of any effects of preexposure. In Experiment 1A, the reinforcer was a lemon-flavored maltodextrin solution, whereas in Experiment 1B, it was a quinine-flavored sucrose solution.

\section{Method}

Subjects and Apparatus. Sixteen naive adult male Wistar rats at least 90 days of age were used in each experiment. They were housed in groups of 4 in transparent plastic cages $(42 \mathrm{~cm}$ long $\times$ $27 \mathrm{~cm}$ wide $\times 17 \mathrm{~cm}$ high). Throughout the preexposure stage and the rest of the experiment, food was freely available in the home cages. The experiments were conducted at the same time each day during the light portion of a 12:12-h light:dark cycle.

Training and testing took place in four identical operant chambers (Letica, S.A.) housed in sound- and light-resistant shells. Each chamber was equipped with a dipper that could deliver $0.05 \mathrm{ml}$ of a $10 \%$ solution of maltodextrin, a complex polysaccharide, flavored with $3 \%$ lemon juice, or a $20 \%$ sucrose solution flavored with $0.0065 \%$ quinine hydrochloride. Both reinforcers were delivered into a recessed magazine that the rats could reach through a flap door positioned in the center of the front wall. A retractable lever was located to the left of this flap door. Each chamber was illuminated by a 7-W, 24-V houselight mounted in the center of the rear panel. An IBM microprocessor located in an adjoining room controlled the equipment and recorded the leverpresses during instrumental training and testing.

The preexposure phase of these experiments was conducted in plastic cages, distinct from the home cages, located in another room that was illuminated by a $60-\mathrm{W}$ white bulb. These cages were opaque plastic boxes ( $25 \mathrm{~cm}$ long $\times 24 \mathrm{~cm}$ wide $\times 15 \mathrm{~cm}$ high) with wire mesh ceilings. They were fitted with calibrated centrifuge tubes from which fluids were delivered through a metal drinking spout inserted in the middle part of the wire mesh.

Procedure. During the preexposure stage of both experiments, all animals were placed on an alternating schedule of low (free access) and high (22.5-h) levels of water deprivation for 6 days. Half of the subjects in each experiment, Groups Low/Low and Low/High, were given preexposure to the solutions that would act as the instrumental reinforcer on Days 1,3 , and 5 , while they were under the low level of water deprivation. Immediately before the start of these sessions, the water was withdrawn from the home cages and the preexposure session began. Four hours after these sessions, these animals were given 1.5 -h access to water in the home cages before it was removed. In this way the subjects were thirsty on the next day. The remaining subjects, Groups High/High and High/Low, received identical preexposure to the reinforcers when water deprived on Days 2, 4, and 6. Four hours after each of these sessions, the animals in these groups received free access to tap water in their home cages. During the preexposure sessions, the animals were placed in the preexposure cages fitted with drinking tubes and were removed 30 min later. For animals in Experiment $1 \mathrm{~A}$, the drinking tubes contained the lemon-flavored maltodextrin solution, whereas for the 
animals in Experiment $1 \mathrm{~B}$, the drinking tubes contained the quinine-flavored sucrose solution. Four hours after the final preexposure session, all animals were returned to free food and water in the home cages and maintained under these conditions throughout instrumental training, which began the next day. Thus, training occurred in the low-deprivation condition.

The instrumental sessions were the same in both experiments, except that in Experiment $1 \mathrm{~A}$, the reinforcer was the maltodextrin solution, whereas in Experiment 1B, it was the sucrose-quinine solution. Animals initially received two 30-min sessions of magazine training in each of which the appropriate reinforcer was delivered on a variable time (VT) 60 -sec schedule with the levers retracted. All animals then received three instrumental training sessions in which leverpressing was reinforced on a variable interval (VI) schedule whose parameter was increased from 5 through 15 to $30 \mathrm{sec}$ across successive sessions. After one further session on the VI 30sec schedule, training was concluded. Each training session started with the insertion of the lever and ended with its retraction $30 \mathrm{~min}$ later. During training, 1 animal in Experiment $1 \mathrm{~A}$ and 3 animals in Experiment $1 \mathrm{~B}$ failed to learn to press the lever, and they were discarded from the experiment.

Immediately after the final training session, all subjects were returned to the alternating schedule of low and high levels of water deprivation for 2 days. During these sessions, the animals received two further exposures to the solutions with the same procedure used during the preexposure phase. Immediately after these sessions, the subjects in Groups High/Low and Low/Low were returned to the freewater schedule. The subjects in the other groups, Groups High/High and Low/High, were maintained on the 22.5 -h water deprivation schedule. The effect of this posttraining shift in primary motivation was assessed in an extinction session on the next day, during which no reinforcers were presented. The test session started with the insertion of the levers and ended with their retraction after $20 \mathrm{~min}$.

Finally, to assess the effectiveness of the posttraining shift in water deprivation on instrumental performance in the presence of the reinforcers, a final 30-min reacquisition session was conducted under the test deprivation state, in which leverpressing was again reinforced with the appropriate reinforcer on a VI 30 -sec schedule.

Throughout this series of experiments, an alpha level of $.05 \mathrm{de}-$ fined statistical significance.

\section{Results and Discussion}

Initially, a combined analysis of the data from the extinction tests in Experiments $1 \mathrm{~A}$ and $1 \mathrm{~B}$ was run to assess the effect of using maltodextrin and sucrose as the instrumental reinforcers. This analysis revealed no effect of experiment $[F(1,20)=1.38]$ nor any significant interaction between this factor and the effects of the test deprivation condition and the preexposure condition $(F \mathrm{~s}<1)$. Consequently, the data from Experiments $1 \mathrm{~A}$ and $1 \mathrm{~B}$ were combined for presentation and analysis, which was conducted on four groups: High/High $(n=7)$, Low/High $(n=7)$, High/Low $(n=7)$, and Low/Low $(n=7)$.

The results from the extinction test and reacquisition session are presented in Figure 1. The left-hand panel of this figure displays the data of primary interest - the number of responses per minute performed during the extinction test in each of the various groups. As shown in this panel, the performance of the animals tested under the low-deprivation state was unaffected by the level of deprivation employed during preexposure to the reinforcer, although Group High/Low tended to press more than Group Low/Low. More importantly, in the absence of pre- exposure to the reinforcer in the high-deprivation state (Group Low/High), a shift to this state in testing did not increase leverpressing in relation to the performance of the animals tested when they were sated (Group Low/Low). Finally, the fact that animals in Group High/High, which were both tested and preexposed under thirst, pressed more than any of the other groups, indicates that sensitivity of instrumental performance to an upshift in water deprivation depends on prior exposure to the reinforcer in the high-deprivation state.

The preceding description of the data was supported by the statistical analysis. We used analysis of covariance to assess possible effects of the baseline performance on the extinction test. The number of responses per minute during the last training session was used as the covariate, with the test deprivation state and the preexposure state as the independent variables. There was no significant effect of the covariate $(F<1)$, indicating that any difference obtained on the extinction test was not influenced by baseline differences between the groups during the training phase. The mean numbers of leverpresses per minute during the final instrumental training session for the various groups were as follows: Group High/High, 6.2; Group Low/High, 4.2; Group High/Low, 5.5; and Group Low/Low, 5.5. The analysis also revealed significant main effects of both deprivation $[F(1,23)=34.77]$ and preexposure $[F(1,23)=$ $5.60]$, and, more important, a significant interaction between these factors $[F(1,23)=4.58]$. This effect was further examined using a series of preplanned orthogonal contrasts based on the predictions derived from the study by López et al. (1992). Neither the difference between Groups High/Low and Low/Low $[F(1,24)=3.20]$ nor the difference between either of these groups and Group Low/ High $[F \mathrm{~s}(1,24) \leq 1.13]$ was significant. By contrast, the difference between Group High/High and the remaining three groups was reliable $[F(1,24)=5.36]$.

It might be argued that the preexposure to the reinforcer while in the high level of deprivation may have increased its reinforcing properties, an effect that might in itself increase extinction performance. The reacquisition data presented in the right panel of Figure 1, however, argue against this possibility. Performance in the groups tested under high deprivation in the final reacquisition session suggests that the instrumental reinforcer was equally effective in the High/High and the Low/High groups. In fact, it is clear from Figure 1 that the subjects in the high-deprivation groups pressed the lever in this session at a substantially higher rate than the animals in the low-deprivation groups. The analysis of leverpress performance during this session, using deprivation and preexposure as the factors, yielded a significant effect of deprivation $[F(1,24)=6.08]$, but no effect of preexposure $[F(1,24)=1.37]$ and no interaction between these factors $(F<1)$.

In summary, these experiments replicate for an increase in water deprivation the pattern of results found by López et al. (1992) for the opposite shift-that is, where rats were trained in a high-deprivation state and tested in 


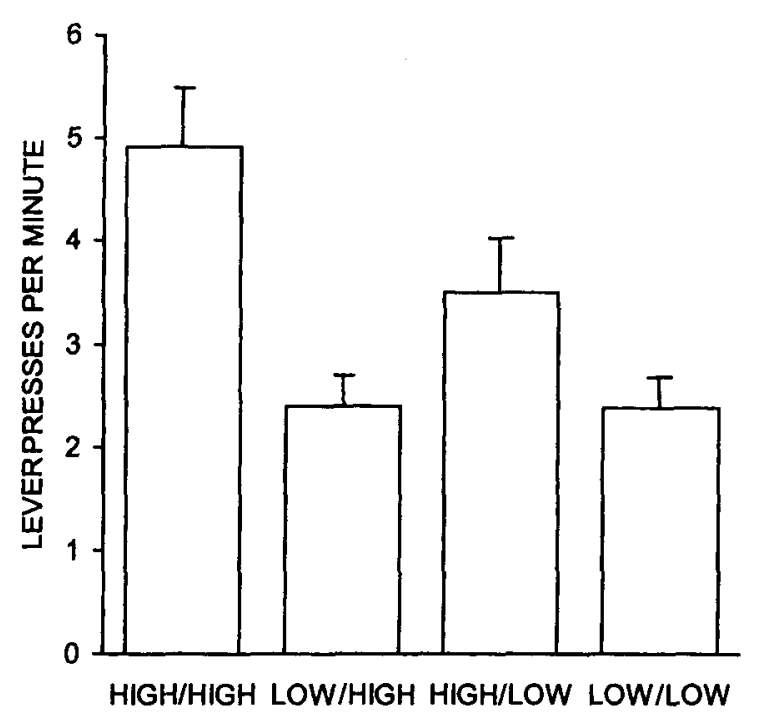

GROUPS

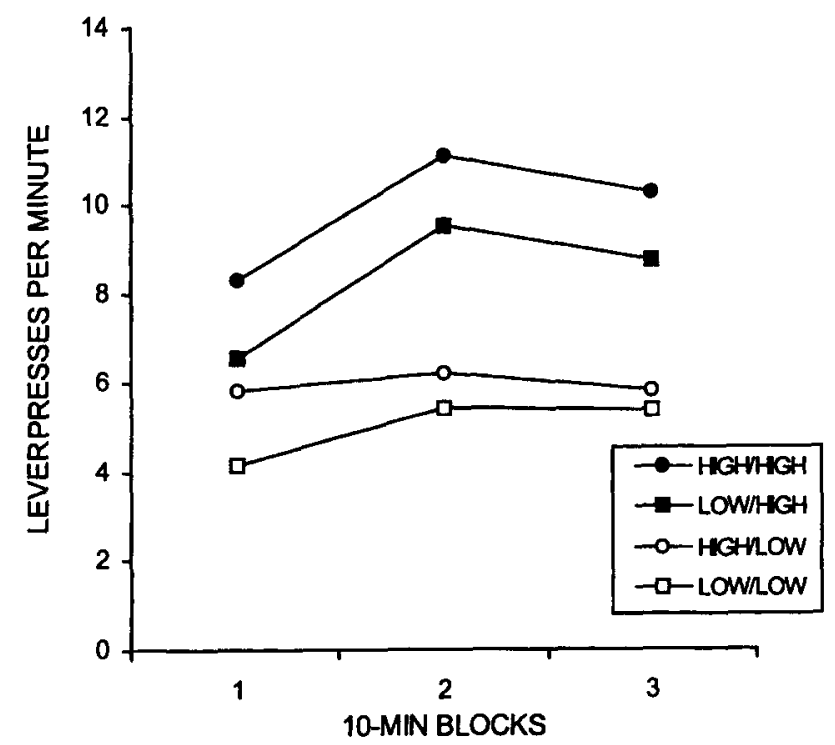

Figure 1. Experiments $1 \mathrm{~A}$ and $1 \mathrm{~B}$ : Mean number of leverpresses per minute during the extinction test session (left panel), and during the reacquisition test in 10-min blocks (right panel), for each of the groups. The first term in each group's label refers to the deprivation state during the preexposure phase and the second one refers to the test deprivation state. Error bars represent the standard errors of the mean.

a low-deprivation state. In this case, rats reduced their leverpress performance only when undeprived if they were allowed to consume the instrumental reinforcer in the low-deprivation state prior to the test. In addition, these experiments replicate for a shift in level of water deprivation the results observed by Balleine (1992) for a comparable transition between levels of food deprivation. Thus, it is clear that animals have to learn about changes in the incentive value of an instrumental outcome through consummatory contact with that outcome in the test motivational state before this change affects instrumental performance.

\section{EXPERIMENT 2}

According to the incentive learning account, the manipulations of reinforcer value produced either through shifts in motivational state or through aversion conditioning should depend on the instrumental contingency experienced during training (Dickinson, 1989; Dickinson \& Balleine, 1994). Although the results of Experiments 1A and $1 \mathrm{~B}$ accord with this claim, the training procedure employed in these studies exposed the animals not only to the instrumental contingency between leverpressing and the reinforcer but also to a Pavlovian relationship between the contextual cues of the conditioning environment and the reinforcer. It has been suggested that the performance of an instrumental action can be influenced by the conditioned affective arousal produced by Pavlovian conditioning (Rescorla \& Solomon, 1967), and there is recent evidence that Pavlovian cues can affect the rate of instrumental performance during an extinction test (e.g., Balleine, 1994). Thus, it would be reasonable to suppose that the preexposure effect observed in the current situation may have been influenced by the conditioned arousing effects of contextual stimuli associated with the instrumental outcome through the repeated presentation of the outcome in the presence of those cues during training.

To assess whether the incentive learning effect obtained in Experiments $1 \mathrm{~A}$ and $1 \mathrm{~B}$ was mediated by the instrumental contingency, in the present experiment, rats were trained to make two responses, each earning a particular outcome (either sucrose or maltodextrin), in separate sessions. Again, as in Experiments 1A and 1B, animals were initially exposed to an alternating schedule of high and low levels of water deprivation. During this stage, all subjects received preexposure to either sucrose or maltodextrin while in a high-deprivation state and to the other reinforcer when in a low-deprivation state. After preexposure animals were maintained on free water and trained to press two levers (counterbalanced as left and right lever), with sucrose delivery contingent on one lever and maltodextrin on the other lever. After this training was complete, all animals were shifted to the high-deprivation state and a test session provided a choice between the two levers in extinction. In this study, the incentive learning account predicts that the preexposure phase, by influencing the incentive values assigned to the reinforcers, will produce a relative increase in performance of the action trained with the reinforcer preexposed in the highdeprivation state when extinction test is conducted in that state. In contrast, an account of the preexposure effect that appeals to the modulatory influence of Pavlovian stimuli 
on instrumental performance would anticipate that the performance of the two actions should not differ in extinction following a shift to the high-deprivation state because the contextual cues were equally associated with both reinforcers during training.

\section{Method}

Subjects and Apparatus. The subjects were 16 male Wistar rats housed under the same conditions as in the previous experiments and trained in the same chambers. In this study, however, each chamber was equipped with two levers positioned symmetrically to the right and left sides of the magazine flap door, respectively. The reinforcers used and their method of delivery were the same as in Experiments $1 \mathrm{~A}$ and $1 \mathrm{~B}$, as were the plastic cages used during the preexposure phase.

Procedure. This experiment used a within-subjects design. The procedure followed that of the first two studies except that the animals received training with two levers and two different reinforcers. Because it is difficult to train the rats when undeprived, we modified the procedure by including a series of instrumental pretraining sessions with the animals placed on a 22.5 -h food deprivation schedule. Initially, animals were magazine trained with food pellets delivered into the magazine on a VT 60-sec schedule with the levers retracted. All animals then received two instrumental pretraining sessions in which leverpressing was reinforced with food pellets on a VI 5-sec schedule. Each session started with the insertion of either the right or the left lever until the animals obtained 15 food pellets. Then, the position of the lever was shifted until they obtained another 15 food pellets. All animals successfully acquired leverpressing when they were trained with food pellets. Following the pretraining sessions, the animals were given free access to food and water for $24 \mathrm{~h}$ in the home cages.

As in the previous studies, all subjects then received a preexposure phase to the incentives with deprivation states alternating daily. During this phase, which lasted 4 days, the animals were placed in the preexposure cages containing the drinking tubes and were given access to the appropriate solution for $30 \mathrm{~min}$. On Days 1 and 3, while in the high-deprivation state, half of the animals received the quinine-flavored sucrose solution, whereas the remaining animals were preexposed to the lemon-flavored maltodextrin solution. Four hours after the completion of these sessions, all subjects were given free access to water in their home cages. By contrast, on Days 2 and 4 , while in the low-deprivation state, all animals received exposure to the alternative solution to the one to which they had been exposed while thirsty. Following the final preexposure session, all subjects were maintained on free water, and the next day instrumental training started. As in Experiments $1 \mathrm{~A}$ and $1 \mathrm{~B}$, training occurred in the low-deprivation state.

During training, the two instrumental responses were rewarded separately on a VI schedule with a parameter that increased from 2 through 5 and $15 \mathrm{sec}$ to $30 \mathrm{sec}$ over successive sessions. After four further sessions on the VI 30-sec schedule, training was concluded. Each session started with the insertion of either the right or left lever until animals obtained 15 reinforcers. After an interval, the other lever was inserted and the session continued until 15 of the other reinforcers had been delivered, at which point it ended. Each action produced a unique outcome either liquid sucrose or the maltodextrin solution. In this way, for half of the animals, pressing the right lever was reinforced with the sucrose and pressing the left lever was reinforced with maltodextrin, with the other animals receiving the opposite action-reinforcer assignment. Moreover, half of the subjects assigned to each action-reinforcer arrangement had been preexposed to the sucrose solution in the high-deprivation state and to the maltodextrin solution in the low-deprivation state, whereas the remaining animals had received the opposite preexposure treatments. Six rats failed to press the two levers and were discarded from the experiment. After this training was complete, the remaining animals received two further exposures to the reinforcers following the procedure used during the preexposure phase.

In a subsequent test, the animals were given a choice between the two response alternatives in the high-deprivation state, but with no outcomes ensuing. The test session started with the insertion of the levers and ended with their retraction after $30 \mathrm{~min}$.

\section{Results and Discussion}

The leverpress performance during training and testing was evaluated by a three-way mixed analysis of variance. Two between-subjects factors, reinforcer and action, distinguished between the reinforcer preexposed in the high-deprivation state and between which action was reinforced by that incentive during training, respectively. The within-subjects preexposure factor distinguished between performance on the action reinforced by the incentive preexposed in the high-deprivation state from that on the other action. An analysis of leverpress performance during the final training session revealed no significant effects of reinforcer and action, nor a significant interaction between these factors $[F(1,6)<2.41$, in all cases]. Furthermore, the difference on the within-subjects factor just failed to reach significance $[F(1,6)=$ 4.58]. The mean numbers of leverpresses during this session for the action reinforced with the incentive preexposed in the high-deprivation state and for the action reinforced with the incentive preexposed in the lowdeprivation state were 36 and 42.4, respectively.

The results of the extinction test are illustrated in Figure 2, which is divided into blocks of $10 \mathrm{~min}$. As this figure suggests, although the difference between the performance of the two actions is small, performance of the action trained with the incentive preexposed in the highdeprivation state was consistently greater than the action previously reinforced with the incentive preexposed in the low-deprivation state. This description of the data was confirmed by the statistical analysis conducted on the number of responses per minute during the extinction test. The analysis revealed a significant effect of the preexposure factor $[F(1,6)=7.57]$, but no effect of the reinforcer or action factors $[F \mathrm{~s}(1,6) \leq 1.97]$. There were no significant interactions between the within-subjects factor and the reinforcer or the action factors $[F(1,6)<1.25$, in each case].

In conclusion, the pattern of results obtained in this experiment confirms the predictions derived from incentive learning theory. The effect on instrumental performance of a revaluation of the reinforcer by a posttraining upshift in the level of thirst was found to depend on exposure to the instrumental reinforcer in the test motivational state prior to the test. Moreover, the present experiment was able to confirm that this incentive learning effect was mediated by the instrumental contingency in force during training. It was found that animals performed more of 


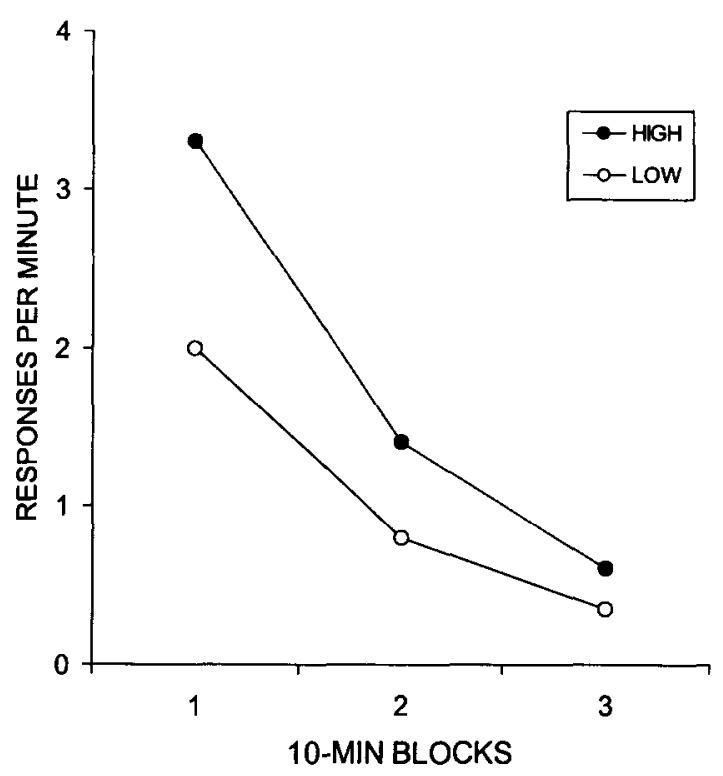

Figure 2. Experiment 2: Mean number of responses per minute on the right- and the left-hand levers during the extinction test, divided by the number of responses reinforced with the incentive preexposed in the high-deprivation state (HIGH) and by the number of responses reinforced with the incentive preexposed in the low-deprivation state (LOW). (Performance is presented averaged across 10-min blocks.)

the action that, in training, had delivered the outcome preexposed in the high water-deprivation state prior to the test than the other action.

\section{GENERAL DISCUSSION}

The results of these experiments support the conclusion that the performance of an instrumental action following a posttraining increase in water deprivation is determined by both the animal's knowledge of the outcome contingent on that action and the current incentive value that the animal assigns to the outcome, a value that is determined by a process of incentive learning. More specifically, Experiments $1 \mathrm{~A}$ and $1 \mathrm{~B}$ demonstrated that, in the absence of an incentive learning treatment, instrumental performance was not affected by a posttraining shift from a low to a high level of water deprivation. If, however, animals were allowed to consume the instrumental outcome in the test deprivation state prior to training, thereby giving them the opportunity to learn about the reinforcer's incentive value in that deprivation state, their performance altered appropriately when shifted to that state following training. Experiment 2, in which rats were trained to perform two leverpress actions prior to a choice extinction test, demonstrated that sensitivity of instrumental performance to a posttraining upshift in the level of water deprivation depends on the instrumental contingency rather than the Pavlovian association between contextual cues and the reinforcer. The preexposure experience with the outcomes produced a relative increase in performance of the action reinforced with the incentive preexposed in the high-deprivation state when the choice test was conducted in that state. These findings offer consistent evidence in support of incentive learning theory and suggest not only that animals learn about the action-reinforcer contingency during instrumental training but also that they learn about the value of the consequences of their actions and that this value can control instrumental performance.

The present experiments serve to extend our knowledge of the generality of incentive learning process to another means of revaluing the instrumental outcome that induced by a posttraining upshift in water deprivation. This role of incentive learning in instrumental performance has been confirmed for a number of different motivational systems and in a number of devaluation paradigms. For example, in addition to its role in revaluation by conditioned taste aversion (Balleine \& Dickinson, 1991, 1992; Balleine, Garner, \& Dickinson, 1995), incentive learning contributes to the control of instrumental performance by shifts in the degree of food deprivation (Balleine, 1992; Balleine \& Dickinson, 1994), shifts from water deprivation to satiety (López et al., 1992), changes in the value of thermoregulation (Hendersen \& Graham, 1979) and sexual (Everitt \& Stacey, 1987) incentives, when outcomes are revalued by drug treatments (Balleine, Ball, \& Dickinson, 1994; Balleine, Davies, \& Dickinson, 1995), and, more recently demonstrated, by specific satiety treatments (Balleine \& Dickinson, 1998). In all these cases it is clear that animals have to learn about their changed affective reactions to the outcome through consummatory contact with that outcome before this change will affect instrumental performance.

It remains to be considered how an upshift in water deprivation acts to cause changes in the incentive value of an instrumental reinforcer. One possible mechanism may be derived from Garcia's (1989) account that the direct experience with a reinforcer in a particular motivational state affects performance by allowing the animal to learn about its affective reactions to the reinforcer in that state. What this account suggests is that, in the absence of consummatory contact with the reinforcer in the high-deprivation state prior to the test, performance will remain unaffected by a shift from a low to a high level of deprivation. Indeed, instrumental performance in Experiments $1 \mathrm{~A}$ and $1 \mathrm{~B}$ was insensitive, in the absence of incentive-learning treatment, to a posttraining upshift in deprivation level. This fact suggests that the increase in performance is based on a relative enhancement of the affective response to the reinforcer consumed in the high-deprivation state. Nevertheless, for each of our results, an alternative explanation could be offered. It is well established that water deprivation reduces food intake, which can result in a nutritional deficit. Throughout our studies, the rats were given exposure to nutritive solutions on days on which they were thirsty, an experience that may well allow them to learn about the nutritive content of the reinforcers. Thus, it is possible that the incentive learning effects observed in the 
present studies are mediated by the nutritive rather than hydrational properties of the solutions employed as reinforcers. There is evidence that a flavor consumed under high deprivation was preferred later to a flavor consumed under low deprivation even when the rats were tested under low deprivation (Revusky, 1967). However, the reacquisition data from Experiments $1 \mathrm{~A}$ and $1 \mathrm{~B}$ argue against the possibility that the nutritive content of the reinforcers may have determined their incentive value because the performance in the groups tested under low deprivation was reduced relative to that of the groups tested under high deprivation. In addition, the operation of such a mechanism in the present experiments seems unlikely because this preference effect has been demonstrated only when the flavors were given separately from feeding in unsweetened food (Capaldi, Sheffer, \& Owens, 1991). Capaldi and her colleagues found that rats preferred the flavor experienced under the higher deprivation condition when unsweetened food was used to deliver the flavors, but the use of sweetened food eliminated this preference. Whatever the merits of the latter account, our data suggest that the reinforcing effectiveness of a nutritive solution does not increase with increasing deprivation, but its hedonic value may increase.

It is of some interest to note that there is independent evidence to suggest that an instrumental action can be sensitive to a change in the value of its outcome in the absence of experience with the revalued outcome. For example, in an experiment by Shipley and Colwill (1996) using a choice procedure, animals trained to make one response for food pellets and a different response for a sucrose solution when hungry showed a preference for the sucrose-trained response in an extinction test conducted under thirst but not in a test conducted under hunger. In a subsequent experiment, these authors found that exposure to sucrose under thirst before testing did not increase the preference for the sucrose response in that deprivation state. Although these findings argue against the incentive learning theory, an alternative explanation of these data could be offered. In these experiments, water was freely available to the animals in their home cage. Consequently, they had repeated opportunity to learn about the high incentive value of water under thirst. To the degree that water and the $8 \%$ sucrose solution used in these studies were not completely discriminated, a direct effect of revaluation of the sucrose outcome on instrumental performance could have been mediated by generalization from the learned value of water under thirst.

Finally, there is additional evidence from a place preference task suggesting that a manipulation in reinforcer value, either through a shift in motivational state or through aversion conditioning, can directly affect performance in this situation. In an experiment by Perks and Clifton (1996), rats exhibited an appropriate preference for a place previously paired with either food or fluid depending on their current motivational state (hunger or thirst). This preference was independent of the animal's prior exposure to the relevant reinforcer in that motiva- tional state. In another study, Perks and Clifton examined the extent to which a sucrose-based place preference is open to manipulation following devaluation of the reinforcer using toxicosis. They found that reinforcer devaluation produces a reduction in place preference even in the absence of the opportunity to revalue the reinforcer following aversion conditioning. These results indicate that performance on a place preference task is directly sensitive to both shifts in motivational state and reduction in reinforcer value. Nevertheless, it should be noted that the findings concerning the absence of a need for incentive learning in this situation are consistent with the argument that incentive learning is not required in a task in which performance is primarily determined by Pavlovian associations. It is possible that performance on a place preference task may be influenced by the association between the contextual stimuli and the outcome. There is evidence to suggest that aversion conditioning produces an immediate reduction in place preference in a similar way to the approach to a food source, such as that involved in magazine entry, following reinforcer devaluation (Balleine, 1992; Balleine \& Dickinson, 1991).

In conclusion, the present experiments demonstrate that instrumental actions are not sensitive to shifts in the value of their outcomes in the absence of consummatory experience with those changed values (i.e., incentive learning). Therefore, our results offer consistent evidence in support of the principles first articulated by Tolman $(1949 \mathrm{a}, 1949 \mathrm{~b})$ and later developed by Dickinson and his colleagues (see Dickinson, 1989, 1997; Dickinson \& Balleine, 1994), for the impact of drive shifts on instrumental performance.

\section{REFERENCES}

BALLEINE, B. W. (1992). Instrumental performance following a shift in primary motivation depends on incentive learning. Journal of Experimental Psychology: Animal Behavior Processes, 18, 236-250.

BaLleine, B. W. (1994). Asymmetrical interactions between thirst and hunger in Pavlovian-instrumental transfer. Quarterly Journal of Experimental Psychology, 47B, 211-231.

Balleine, B. W., Ball, J., \& Dickinson, A. (1994). Benzodiazepineinduced outcome revaluation and the motivational control of instrumental action in rats. Behavioral Neuroscience, 108, 573-589.

Balleine, B. W., Davies, A., \& Dickinson, A. (1995). Cholecystokinin attenuates incentive learning in rats. Behavioral Neuroscience, 109, 312-319.

BALleine, B. W., \& Dickinson, A. (1991). Instrumental performance following reinforcer devaluation depends on incentive learning. Quarterly Journal of Experimental Psychology, 43B, 279-296.

Balleine, B. W., \& Dickinson, A. (1992). Signalling and incentive processes in instrumental reinforcer devaluation. Quarterly Journal of Experimental Psychology, 45B, 285-301.

Balleine, B. W., \& Dickinson, A. (1994). The role of cholecystokinin in the motivational control of instrumental action. Behavioral Neuroscience, 108, 590-605.

Balleine, B. W., \& Dickinson, A. (1998). The role of incentive learning in instrumental outcome revaluation by sensory-specific satiety. Animal Learning \& Behavior, 26, 46-59.

Balleine, B. W., Garner, C., \& Dickinson, A. (1995). Instrumental outcome-devaluation is attenuated by the anti-emetic ondansetron. Quarterly Journal of Experimental Psychology, 48B, 235-251.

CAPALDI, E. D., SheFFER, J. D., \& OWENS, J. (1991). Food deprivation 
and conditioned flavor preferences based on sweetened and unsweetened foods. Animal Learning \& Behavior, 19, 361-368.

DICKINSON, A. (1989). Expectancy theory in animal conditioning. In S. B. Klein \& R. R. Mowrer (Eds.), Contemporary learning theories: Pavlovian conditioning and the status of traditional learning theory (pp. 279-308). Hillsdale, NJ: Erlbaum.

DiCKINSON, A. (1997). Bolle's psychological syllogism. In M. E. Bouton \& M. S. Fanselow (Eds.), Learning, motivation, and cognition. The functional behaviorism of Robert C. Bolles (pp. 345-367). Washington, DC: American Psychological Association.

Dickinson, A., \& Balleine, B. W. (1994). Motivational control of goal-directed action. Animal Learning \& Behavior, 22, 1-18.

Everitt, B. J., \& STACEY, P. (1987). Studies of instrumental behavior with sexual reinforcement in male rats (Rattus norvegicus): 2 . Effects of preoptic area lesions, castration, and testosterone. Journal of Comparative Psychology, 101, 407-419.

GarCIA, J. (1989). Food for Tolman: Cognition and cathexis in concert. In T. Archer \& L.-G. Nilsson (Eds.), Aversion, avoidance and anxiety (pp. 45-85). Hillsdale, NJ: Erlbaum.

Hendersen, R. W., \& Graham, J. (1979). Avoidance of heat by rats: Effects of thermal context on the rapidity of extinction. Learning \& Motivation, 10, 351-363.
Hull, C. L. (1943). Principles of hehavior. New York: Appleton-CenturyCrofts.

LóPEZ, M., BALleine, B. W., \& Dickinson, A. (1992). Incentive learning and the motivational control of instrumental performance by thirst. Animal Learning \& Behavior, 20, 322-328.

Perks, S. M., \& Clifton, P. G. (1996). Reinforcer devaluation and conditioned place preference. Physiology \& Behavior, 61, 1-5.

Rescorla, R. A., \& Solomon, R. L. (1967). Two-process learning theory: Relationship between Pavlovian conditioning and instrumental learning. Psychological Review, 74, 151-182.

REvUSKY, S. H. (1967). Hunger level during food consumption: Effects on subsequent preference. Psychonomic Science, 7, 109-110.

Shipley, B. E., \& Colwill, R. M. (1996). Direct effects on instrumental performance of outcome revaluation by drive shifts. Animal Learning \& Behavior, 24, 57-67.

Tolman, E. C. (1949a). The nature and functioning of wants. Psychological Review, 56, 357-369.

Tolman, E. C. (1949b). There is more than one kind of learning. Psychological Review, 56, 144-155.

(Manuscript received August 6, 1998; revision accepted for publication March 2, 1999.) 